\title{
Total Surface Current Vectors and shear from a sequence of satellite images. Part 1: effect of waves in opposite directions
}

\author{
Fabrice Ardhuin ${ }^{1,2}$ and Matias Alday ${ }^{1}$ \\ Maria Yurovskaya ${ }^{3}$ \\ ${ }^{1}$ Univ. Brest, CNRS, Ifremer, IRD, Laboratoire d'Océanographie Physique et Spatiale, Brest, France \\ ${ }^{2}$ Scripps Institution of Oceanography, University of California San Diego, La Jolla, California \\ ${ }^{3}$ Marine Hydrophysical Institute of RAS, Sevastopol, Russia \\ Key Points: \\ - Near surface current shear are given by phase speeds derived from lagged pairs \\ of satellite images \\ - Waves in opposite directions are the main source of errors for the shortest wave \\ components \\ - At least 3 images are required to separate waves in opposite directions
}

Corresponding author: Fabrice Ardhuin, ardhuin@ifremer.fr 


\begin{abstract}
The Total Surface Current Velocity (TSCV) is the horizontal vector quantity that advects seawater, and it is an Essential Climate Variable that has been difficult to estimate from space. Following ground-based techniques, the TSCV can be derived from the phase speed of surface gravity waves, and the different estimates for different wavelengths are indicative of vertical shear that is of great interest. Here we combine satellite imagery and numerical simulation of the images to investigate the conditions in which shear could be measured. Using 10-m resolution images from the Level-1c Red Green and Blue channels of the Sentinel 2 Multispectral Instrument, acquired with time lags of $0.5 \mathrm{~s}$, it is found that retrieving the near surface shear requires a specific attention to waves in opposing direction. Implementing a least-square fit in Fourier space of the current speed and amplitudes of waves in opposing directions to the observed complex amplitudes of a sequence of 3 images, the phase speed and current can be estimated much more accurately than with only a pair of images, down to the Nyquist wavelength of $20 \mathrm{~m}$. A byproduct of this analysis is the "opposition spectrum" that is a key quantity in the sources of microseisms and microbaroms. A sensitivity analysis suggests that the retrieval of TSCV and shear can be improved by increasing time lags, increasing resolution and increasing the exposure time of acquisition, using a push-frame technique instead of MSI's push-broom technique. These finding open the way for new investigations of near-surface ocean processes including regions of freshwater influence and possibly internal waves.
\end{abstract}

\title{
Plain Language Summary
}

Measuring ocean surface current and its vertical variation is important for a wide range of science questions and applications.

\section{Introduction}

Surface current velocities play an important role in may ocean processes, including the flux of kinetic energy from the atmosphere to the ocean, air-sea fluxes (Cronin et al., 2019), and the transport of buoyant material (Maximenko et al., 2019). Many observation systems have been proposed to measure the surface current in a wide range of conditions. (Barrick, 1977) and many others have developed land-based HF radars that rely on the dispersion relation of surface gravity waves, while open ocean conditions are very sparsely monitored by in situ moorings with measurements at depths larger than $5 \mathrm{~m}$, Surface Velocity Program (SVP) drifters with a depth of measurement around $15 \mathrm{~m}$ when their drogue is attached (Niiler \& Paduan, 1995), and a not so clear combination of wind and current speeds in the absence of that drogue, surface drifts of Argo floats, and, for the lack of a better alternative, estimates based on satellite remote sensing of wind, seal level anomalies from altimeters, and a combination of drifters and satelite gravimeters for the Mean Dynamic Topography (Rio et al., 2014).

These different estimates of the near-surface current can be rather large, in part due to the sampling of different depths. It is thus desirable to measure the vertical shear of the current in order to be able to compare these estimates. The shear is also an important indication of mixing or lack thereof, giving information on possible stratification.

Shear estimates have used the wave dispersion modification due to the current vector, defined by the two components $U_{x}(z)$ and $U_{y}(z)$ of the horizontal current profile (Stewart \& Joy, 1974). For completeness, a non-linear wave correction should also be included (Broche et al., 1983), which is almost the same as replacing the Eulerian mean current by the Lagrangian mean current (Andrews \& McIntyre, 1978). We thus expect, for $k D \gg 1$,

$$
U_{a}(k, \varphi)=\int_{-D}^{0} U_{x}(z) \exp (2 k z) \mathrm{d} z \cos \varphi+\int_{-D}^{0} U_{y}(z) \exp (2 k z) \mathrm{d} z \sin \varphi=U_{a} \cos \left(\varphi-\varphi_{U}\right) .
$$




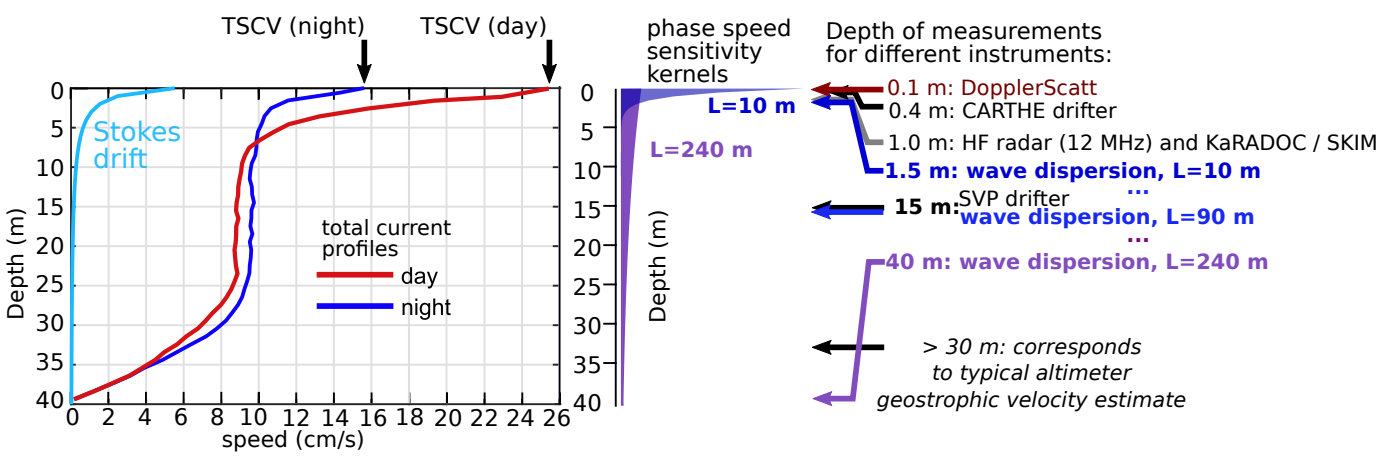

Figure 1. Left: typical day and night velocity profiles of the total current in the Atlantic at $26^{\circ} \mathrm{N}, 36^{\circ} \mathrm{W}$ (adapted from Sutherland et al. 2016). Center: sensitivity kernels for surface gravity wave phase speeds. Right: depth of measurement of different instruments

Obtaining current shear from a sequence of images has been done from many sensors (?, ?; Laxague et al., 2015). It requires to get reliable estimates $U(k, \varphi)$, for different wavelengths, including the shortest components.

A great opportunity is offered by satellite imagery that has been used to estimate surface current (Kudryavtsev et al., 2017b). The objective of the present paper is to discuss the accuracy of those estimates, starting with the 2-image method used by (Kudryavtsev et al., 2017b), which is discussed in section 2. In order to demonstrate the different processing steps and the influence of the image properties, we rely on the comparison of true data and simulated images generated using the simulator described in Appendix A. Due to the possible corruption of phase speeds by waves in opposing directions, we propose a new method in section 3, using sequences of 3 images, with details given in Appendix B. Discussions and conclusions follow in section 4 .

\section{Effect of waves in opposite directions with 2-image method}

We start from the same image example that was used Kudryavtsev et al. (2017b), acquired off the Calfornia coast in the region of San Diego. The image processing method is illustrated in Fig. 2.

In order to understand the processing results, we also have generated simulated images and applied the exact same processing to the simulated images. The image simulator is described in more detail in Appendix A, and corresponds to the forward model of Kudryavtsev et al. (2017a), combined with a noise model. The model input parameters are the Sentinel 2 viewing geometry, an estimate of the surface wind vector given by satellite scatterometer data, and a directional wave spectrum that is estimated from an in situ buoy. The buoy is station number 220 of the Coastal Data Information Program (CDIP) located at $32.752 \mathrm{~N} 117.501 \mathrm{~W}$ and is also identified by the World Meteorological Organization by the number 46258 .

The first 5 directional moments are converted to a 5-degree resolution directional frequency spectrum using the Maximum Entropy Method (Lygre \& Krogstad, 1986). This spectrum is then interpolated onto a regular grid in $\left(k_{x}, k_{y}\right)$ space with a resolution of $1 / 16000$ cycles per meter. Drawing random phases for each spectral component the power spectral density is used to define complex amplitudes that are inverse-Fourier transformed 
to generate $8 \mathrm{~km}$ square grids of the surface elevation and long wave slopes, $\left(s_{x}(x, y), s_{y}(x, y)\right)$, at $10 \mathrm{~m}$ resolution.

The input to our image simulator are thus

- the wave spectrum $E\left(k_{x}, k_{y}\right)$

- the direction of the dominant slopes $\varphi_{\mathrm{mss}}$

- the mean square slope in that direction $\mathrm{mss}_{u}$ and the mean square slope in the perpendicular cross-direction $\mathrm{mss}_{c}$.

- the images bistatic view angles $\beta$ and $\phi^{\prime}$ assumed constant for each image.

The forward model described in Kudryavtsev et al. (2017a) is used to compute a mean luminance $B_{0}$ for a locally rough but flat surface, and the local luminance $B(x, y)$ from the same rough surface tilted by the long wave slopes. Detected luminance fluctuations are caused by the true luminance fluctuations caused by the finite number of specular points (Longuet-Higgins, 1960) that contribute to the signal ... The image pixel value is then taken as the nearest integer of a mean intensity $\langle I\rangle$ times $\left(1+n_{t}\right) B / B_{0}$ where $n_{t}$ is a random white noise of a amplitude $N_{t}$ that parameterizes the "twinkle" of the sea surface

The noise detector is treated as an additive noise $n_{d}$, represented as a Gaussian noise of standard deviation $N_{d}$. For each channel $j$ which corresponds to a time $t_{j}$ we have the pixel value

$$
I_{j}(x, y)=\mathrm{E}\left(\langle I\rangle_{j} B\left(x, y, t_{j}\right) / B_{0}\left(1+n_{t}\right)\right),
$$

where the value $\mathrm{E}(x)$ is the largest integer value that is less or equal to $x$.

The quantization effect of rounding to an integer pixel value is not very relevant in the present paper. However, the noise model does have a very important influence on the estimation of the surface current, as will be discussed below.

Because the shorter wave components have a low coherence due to their large directional spreading, we used a phase estimated from the coherent sum of the complex amplitudes obtained from individual image tiles that are $500 \mathrm{~m}$ wide. We first sum the $16^{2}=256$ tiles, and then add $15^{2}$ tiles that are shifted by $250 \mathrm{~m}$ in each direction in order to use the signal that is otherwise much reduced by the 2-dimension Hanning window. This gives 512 degrees of freedom for each spectral estimate.

The shortest waves that propagate along the $x$ axis in the image have a $20 \mathrm{~m}$ wavelength. Their phase speed, for zero current, is expected to be $5.6 \mathrm{~m} / \mathrm{s}$ and they should be displaced by $0.6 \mathrm{~m}$ between the red and the blue channels that are separated by $1.1 \mathrm{~s}$, and only $0.3 \mathrm{~m}$ between the red and green. Although this distance is much smaller than the $10 \mathrm{~m}$ pixel size, it is easily picked up bu Fourier analysis, and the phase speed of these short and slow waves may actually be retrieved by averaging many Fourier transforms. In fact, Fig. 2 shows that the phase speeds down to $25 \mathrm{~m}$ wavelength are consistent with the theory. However, at $20 \mathrm{~m}$ wavelength there is a "noise" that gives a positive bias to the velocities with an order of magnitude of $3 \mathrm{~m} / \mathrm{s}$ (see dashed magenta circle in 2). Interestingly the same "noise" is also present in the simulated data. Trial and error showed that it was not associated to the amplitude or the additive or multiplicative nature of the noise in eq. (2), as long as some energy remains for waves in opposing directions.

This "noise" disappears in the simulation when the input spectrum is "chopped" to remove westward propagating waves.

\section{NEED TO ADD FIGURE HERE .}

Indeed, any spectral component $(k, \varphi)$ contains information that propagate in both directions $\varphi$ and $\varphi+\pi$, by interpreting the phase difference $\psi_{4,2}$ as the phase of a single travelling wave, we are assuming that we can neglect the waves in direction $\varphi+\pi$. 

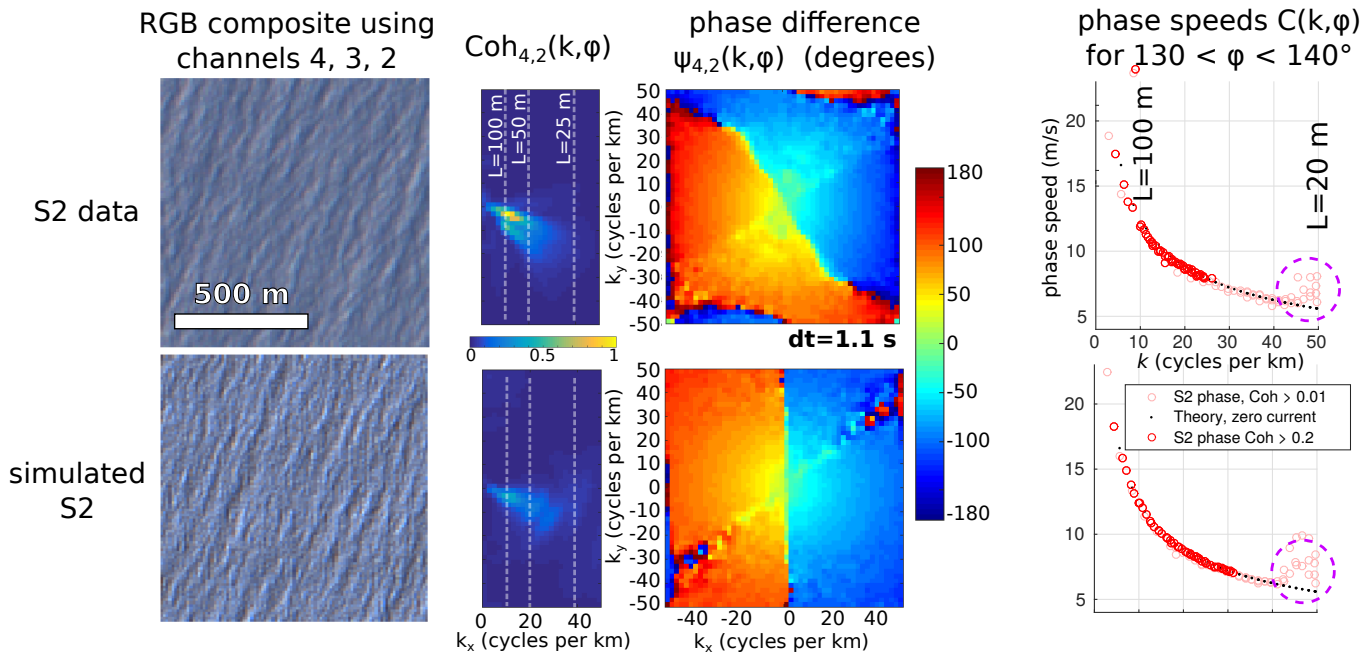

Figure 2. Processing from Level-1c images to phase speeds, using 500 x $500 \mathrm{~m}$ tiles over a 8 by $8 \mathrm{~km}$ area, giving 256 degrees of freedom. Top: data from Copernicus Sentinel 2 on 29 April 2016 off California (See Figs. 3-9 in Kudryavtsev et al. 2017), with $\beta=14^{\circ}, U_{10}=6 \mathrm{~m} / \mathrm{s}$. Middle: simulated S2 data based on in situ wave spectrum determined from directional moments using the Maximum Entropy Method, and with random phases. The noise amplitude is 0.3. The present paper was motivated by the phase speed anomalies, highlighted with the dashed magenta circle near the Nyquist wavelength $L=20 \mathrm{~m}$.

In fact, the data is in general the sum of two waves travelling in these opposite directions, each giving a different phase difference. In order to obtain the three informations that are the 2 amplitudes and one relative phase we need more than 2 images. We have thus developed a method to estimate the current velocity from a sequence of 3 images.

\section{Least squares method applied to a sequence of 3 images}

The details of the method are given in Appendix A. They correspond to the adaptation of the method by Mansard and Funke (1980) for the estimation of the incident and reflected wave amplitudes in a laboratory flume using 3 time series from 3 different wave gauges, with the addition of an unknown current. In fact, eq. (B27) can be applied directly to that problem: knowing the measured complex amplitudes given by the Fourier analysis (in time) of the 3 gauges, one gets the complex amplitude of the incident and reflected waves and the current along the flume, as illustrated in Fig. 3.

For the case of 3 images, the complex amplitudes of the intensities of the 3 images, for any spectral component with wavenumber $k$ and azimuth $\varphi$ are separated into a complex amplitude $Z_{A}(k, \varphi)$ of a wave train travelling in direction $\varphi$ and $Z_{B}(k, \varphi)$ travelling in direction $\varphi+\pi$, and a current $U(k, \varphi)$ that should be equal to $U_{a}(k, \varphi)$ given by eq. (1).

In order to quantify the magnitude of waves in opposing directions, we define an "opposition spectrum",

$$
H(k, \varphi)=2 \frac{\left|Z_{A}\right|^{2}(k, \varphi)\left|Z_{B}\right|^{2}(k, \varphi)}{\left|Z_{A}\right|^{2}+\left|Z_{B}\right|^{2}}
$$

that ranges form 0 for waves propagating only in direction $\varphi$ to 1 for equal amplitudes in opposing directions. This is the spectral analogue of the "overlap integral" $I(k)$ defined by (Farrell \& Munk, 2008) and first used by Hasselmann et al. (1963) for the the- 

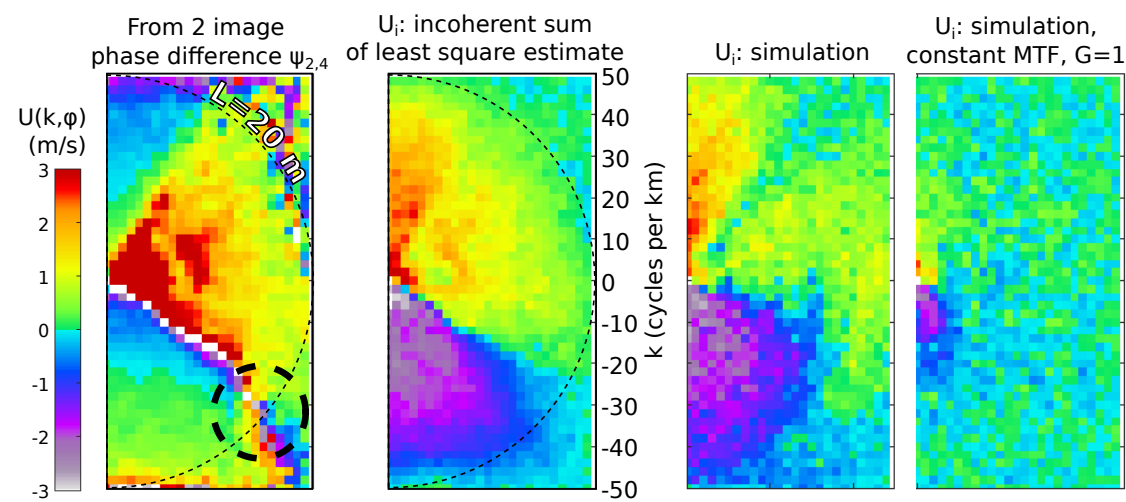

$\mathrm{U}_{\mathrm{i}}$ : simulation,
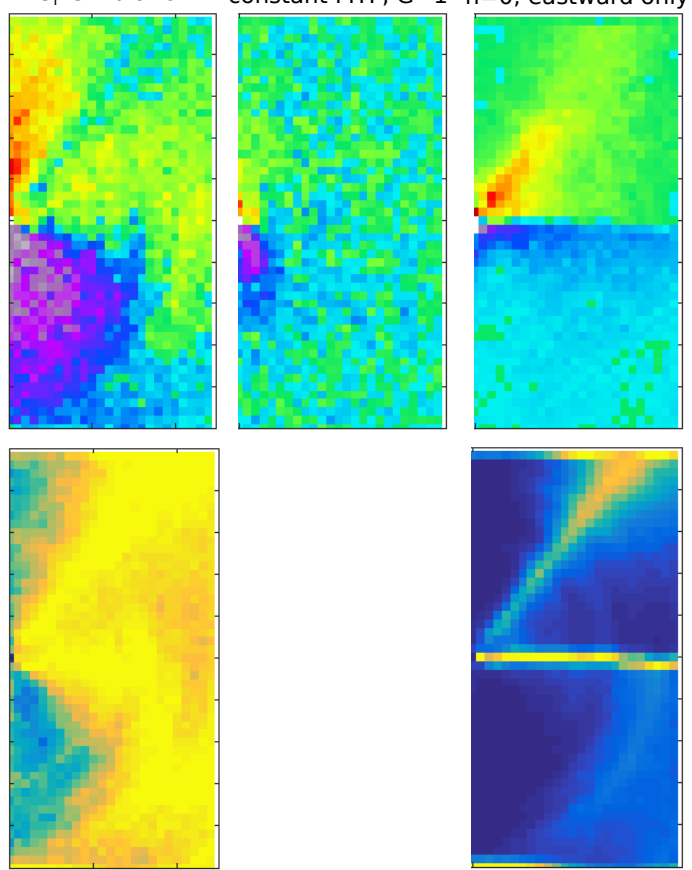

$H(k, \varphi)$
0.25
0.20
0.15
0.10
0.05
0.00

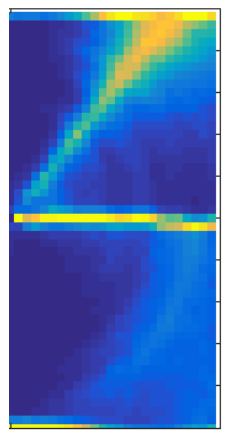

Figure 3. Current and opposition spectra from the S2 image shown in figure 1 using a processing from 2 or 3 image channels.

ory of generation of secondary microseisms and microbaroms (Ardhuin et al., 2015; De Carlo et al., 2020). If $H(k, \varphi)$ is independent of $\varphi$ then $I(k)=H(k, \varphi)$.

We started to make estimations of the current by inverting eq. (B27) for each combination of complex amplitudes from a single FFT tile and averaging over all the tiles, $U_{i}(k, \varphi)$. This incoherent sum happens to be biased by the modulation transfer function (MTF) between the surface elevation and image intensity in the presence of a significant multiplicative noise, as confirmed by the simulations in Fig. 4. The incoherent estimate

Figure 4. Estimation of the current matrix $U_{i}(k, \varphi)$ and overlap matrix $H(k, \varphi)$ using an incoherent average of the velocities obtained from each image tile. (a) derived from the S2 image, (b) simulated S2 image, (c) simulated without noise, (d) simulated without the image MTF.

$U_{i}(k, \varphi)$, with an example in in Fig. 4.a, is a great opportunity for measuring the MTF in the absence of currents, but it is a problem if one wants to estimate currents with a high accuracy and not knowing the MTF.

\section{Discussion and Conclusions}


or

$$
\begin{aligned}
A+B-F_{1} & =\varepsilon_{1} \\
A \mathrm{e}^{-\mathrm{i} \mu-\chi}+B \mathrm{e}^{+\mathrm{i} \mu-\chi}-F_{2} & =\varepsilon_{2} \\
A \mathrm{e}^{-\mathrm{i} \nu-\psi}+B \mathrm{e}^{+\mathrm{i} \nu-\psi}-F_{3} & =\varepsilon_{3}
\end{aligned}
$$

\section{Appendix B Adaptation of 3-probe least squares method to an un-}

Let us have $A$ and $B$ the complex amplitudes of the waves propagating in the $\varphi$ with $U$ the current component in direction $\varphi, \sigma=\sqrt{g k}, \mu=\sigma t_{2}, \nu=\sigma t_{3}, \chi=k U t_{2}$, $\psi=k U t_{3}$

$$
\begin{aligned}
& F_{1}=A+B+N_{1} \\
& F_{2}=A \mathrm{e}^{-\mathrm{i} \mu-\chi}+B \mathrm{e}^{+\mathrm{i} \mu-\chi}+N_{2} \\
& F_{3}=A \mathrm{e}^{-\mathrm{i} \nu-\psi}+B \mathrm{e}^{+\mathrm{i} \nu-\psi}+N_{3}
\end{aligned}
$$

and we look for the solution that minimizes the sum of the $\varepsilon_{n}$ squared,

$$
\sum_{n} \varepsilon_{n}^{2}=\sum_{n}\left(A \mathrm{e}^{-\mathrm{i} \sigma t_{n}-k U t_{n}}+B \mathrm{e}^{+\mathrm{i} \sigma t_{n}-k U t_{n}}-F_{n}\right)^{2}
$$

Taking derivatives with respect to $A, B$ and $U$ gives, respectively,

$$
\begin{array}{rc}
\sum_{n} \mathrm{e}^{-\mathrm{i}\left(\sigma t_{n}-k U t_{n}\right)}\left(A \mathrm{e}^{-\mathrm{i}\left(\sigma t_{n}+k U t_{n}\right)}+B \mathrm{e}^{+\mathrm{i}\left(\sigma t_{n}-k U t_{n}\right)}-F_{n}\right) & =0 \\
\sum_{n} \mathrm{e}^{\mathrm{i}\left(\sigma t_{n}+k U t_{n}\right)}\left(A \mathrm{e}^{-\mathrm{i}\left(\sigma t_{n}+k U t_{n}\right)}+B \mathrm{e}^{+\mathrm{i} \sigma t_{n}-k U t_{n}}-F_{n}\right) & =0 \\
\sum_{n} t_{n}\left(A \mathrm{e}^{-\mathrm{i}\left(\sigma t_{n}+k U t_{n}\right)}+B \mathrm{e}^{\mathrm{i}\left(\sigma t_{n}-k U t_{n}\right)}\right)\left(A \mathrm{e}^{-\mathrm{i}\left(\sigma t_{n}+k U t_{n}\right)}+B \mathrm{e}^{+\mathrm{i}\left(\sigma t_{n}-k U t_{n}\right)}-F_{n}\right) & =0
\end{array}
$$

using $t_{1}=0$, this can be re-arranged as

\section{Appendix A Image simulator}

$$
\begin{aligned}
\alpha_{1} A & +\beta_{1} B=\gamma_{1} \\
\beta_{1} A & +\beta_{2} B=\gamma_{2} \\
t_{2}\left(\alpha_{3} A+\beta_{3} B\right) & \cdot \quad\left(\alpha_{3} A+\beta_{3} B-F_{2}\right) \\
+t_{3}\left(\alpha_{4} A+\beta_{4} B\right) & \cdot \quad\left(\alpha_{4} A+\beta_{4} B-F_{3}\right)=0
\end{aligned}
$$

where we have defined

$$
\begin{aligned}
& \alpha_{1}=\left[1+\mathrm{e}^{-\mathrm{i}(2 \sigma+2 k U) t_{2}}+\mathrm{e}^{-\mathrm{i}(2 \sigma+2 k U) t_{3}}\right] \\
& \beta_{1}=\left[1+\mathrm{e}^{-\mathrm{i} 2 k U t_{2}}+\mathrm{e}^{-\mathrm{i} 2 k U t_{3}}\right] \\
& \gamma_{1}=F_{1}+F_{2} \mathrm{e}^{-\mathrm{i}(\sigma+k U) t_{2}}+F_{3} \mathrm{e}^{-\mathrm{i}(\sigma+k U) t_{3}} \\
& \beta_{2}=\left[1+\mathrm{e}^{2 \mathrm{i}(\sigma-k U) t_{2}}+\mathrm{e}^{2 \mathrm{i}(\sigma-k U) t_{3}}\right]
\end{aligned}
$$




$$
\begin{aligned}
\gamma_{2} & =F_{1}+F_{2} \mathrm{e}^{\mathrm{i}(\sigma-k U) t_{2}}+F_{3} \mathrm{e}^{\mathrm{i}(\sigma-k U) t_{3}} \\
\alpha_{3} & =\mathrm{e}^{\mathrm{e}-\mathrm{i}(\sigma+k U) t_{2}} \\
\beta_{3} & =\mathrm{e}^{\mathrm{i}(\sigma-k U) t_{3}} \\
\alpha_{4} & =\mathrm{e}^{\mathrm{e}-\mathrm{i}(\sigma+k U) t_{3}} \\
\beta_{4} & =\mathrm{e}^{\mathrm{i}(\sigma-k U) t_{3}}
\end{aligned}
$$

We may eliminate $A$ and $B$ from the first 2 equations,

$$
A=\left(r_{F, 1}-r_{B, 1} B\right) / r_{A, 1},
$$

and

$$
B=\left(\gamma_{2}-{ }_{1} \beta_{1} / \alpha_{1}\right) /\left(\beta_{2}-\beta_{1}^{2} / \alpha_{1}\right) .
$$

replacing these expressions for $A$ and $B$ in eq. (B15) gives one equation for $U$,

$$
\begin{array}{rll}
f\left(U, k, \sigma, F_{1}, F_{2}, F_{3}, t_{2}, t_{3}\right)= & \left.t_{2} \quad\left(\alpha_{3} A+\beta_{3} B\right) \times\left(\alpha_{3} A+\beta_{3} B-F_{2}\right)\right) \\
+ & \left.t_{3} \quad\left(\alpha_{4} A+\beta_{4} B\right)\right) \times\left(\alpha_{4} A+\beta_{4} B-F_{3}\right)=0 .
\end{array}
$$

Finding the solution for $f=0$ gives an estimate of the value of $\mathrm{U}$. This operation can be repeated for each Fourier transform (each tile) and each spectral component. Different averaging procedures are discussed in section 3 .

\section{Acknowledgments}

We acknowledge the use of Copernicus Sentinel 2 data, obtained from the Copernicus Science Hub https://scihub.copernicus.eu. F.A. and M.A. were supported by CNES as part of the SKIM preparation program and ANR grants for ISblue (ANR-17-EURE-0015) LabexMER (ANR-10-LABX-19), and MIMOSA (ANR-14-CE01-0012).

\section{References}

Andrews, D. G., \& McIntyre, M. E. (1978). On wave action and its relatives. $J$. Fluid Mech., 89, 647-664. (Corrigendum: vol. 95, p. 796)

Ardhuin, F., Gualtieri, L., \& Stutzmann, E. (2015). How ocean waves rock the earth: two mechanisms explain seismic noise with periods 3 to $300 \mathrm{~s}$. Geophys. Res. Lett., 42, 765-772. doi: 10.1002/2014GL062782

Barrick, D. E. (1977). Extraction of wave parameters from measured HF radar seaecho Doppler spectra. Radio Science, 12, 415-423.

Broche, P., de Maistre, J. C., \& Forget, P. (1983). Mesure par radar décamétrique cohérent des courants superficiels engendrés par le vent. Oceanol. Acta, 6(1), $43-53$.

Cronin, M. F., Gentemann, C. L., Edson, J., Ueki, I., Bourassa, M., Brown, S., ... Zhang, D. (2019). Air-sea fluxes with a focus on heat and momentum. Frontiers in Marine Sci., 6, 430.

De Carlo, M., Ardhuin, F., \& Pichon, A. L. (2020). Atmospheric infrasound radiation from ocean waves in finite depth: a unified generation theory and application to radiation patterns. Geophys. J. Int., 221, 569-585. doi: $10.1093 /$ gji $/$ ggaa015

Farrell, W. E., \& Munk, W. (2008). What do deep sea pressure fluctuations tell about short surface waves? Geophys. Res. Lett., 35(7), L19605. doi: 10.1029/ 2008GL035008

Hasselmann, K., Munk, W., \& MacDonald, G. (1963). Bispectra of ocean waves. In M. Rosenblatt (Ed.), Time series analysis (pp. 125-139). Wiley.

Kudryavtsev, V., Yurovskaya, M., Chapron, B., Collard, F., \& Donlon, C. (2017a). Sun glitter imagery of surface waves. part 1: Directional spectrum retrieval and validation. J. Geophys. Res., 122. doi: 10.1002/2016JC012425 
Kudryavtsev, V., Yurovskaya, M., Chapron, B., Collard, F., \& Donlon, C. (2017b). Sun glitter imagery of surface waves. part 2: Waves transformation on ocean currents. J. Geophys. Res., 122. doi: 10.1002/2016JC012426

Laxague, N. J. M., Özgökmen, T. M., Haus, B. K., Novelli, G., Shcherbina, A., Sutherland, P., ... Molemaker, J. (2015). Observations of near-surface current shear help describe oceanic oil and plastic transport. Geophys. Res. Lett., 44, 245-249. doi: 10.1002/2017GL075891

Longuet-Higgins, M. S. (1960). Reflection and refraction at a random moving surface. II. number of specular points in a gaussian surface. J. Opt. Soc. Am. 50(9), 845-850. doi: 10.1364/JOSA.50.000845

Lygre, A., \& Krogstad, H. E. (1986). Maximum entropy estimation of the directional distribution in ocean wave spectra. J. Phys. Oceanogr., 16, 2,052-2,060.

Mansard, E. P. D., \& Funke, E. R. (1980). The measurement of incident and reflected spectra using a least squares method. In Proceedings of international conference on coastal engineering (Vol. 1). doi: 10.9753/icce.v17.8

Maximenko, N., Corradi, P., Law, K. L., Sebille, E. V., Garaba, S. P., Lampitt, R. S., ... Wilcox, C. (2019). Toward the integrated marine debris observing system. Frontiers in Marine Sci., 6, 447. doi: 10.3389/fmars.2019.00447

Niiler, P. P., \& Paduan, J. D. (1995). Wind-driven motions in the Northeast Pacific as measured by Lagrangian drifters. J. Phys. Oceanogr., 25 (11), 2819-2930. Retrieved from http://ams.allenpress.com/archive/1520-0485/25/11/ pdf/i1520-0485-25-11-2819

Rio, M.-H., Mulet, S., \& Picot, N. (2014). Beyond GOCE for the ocean circulation estimate: Synergetic use of altimetry, gravimetry, and in situ data provides new insight into geostrophic and Ekman currents. Geophys. Res. Lett., 41, 8918-8925. doi: 10.1002/2014GL061773

Stewart, R. H., \& Joy, J. W. (1974). HF radio measurements of surface currents. Deep Sea Res., 21, 1039-1049. 